\title{
Impact of COVID-19 on pediatric oncology
}

\author{
Katarzyna Adamczewska-Wawrzynowicz ${ }^{1^{*}}$, Swati Singh ${ }^{2}$, Katarzyna Derwich ${ }^{1}$ \\ ${ }^{1}$ Department of Pediatric Oncology, Hematology and Transplantology, Poznan University of Medical Sciences, Poznań, Poland \\ ${ }^{2}$ Center for Medical Education in English, Poznan University of Medical Sciences, Poznań, Poland
}

\section{Abstract}

The worldwide coronavirus disease 19 (COVID-19) pandemic led to a critical situation in the healthcare system concerning patients suffering from cancer and chronic diseases. It had the effect of causing them anxiety and reluctance to seek immediate medical attention when required due to the fear of COVID-19's potentially acute and life-threatening states.

The main issues concerning pediatric patients in this regard are the reluctance to seek pediatric care leading to delayed referrals for severe conditions, including children's neoplasms, that show a history of symptoms indicating cancer that has been present for weeks. The risk of prolonging diagnoses of pediatric neoplasms may be incomparably more threatening than the severe acute respiratory syndrome coronavirus 2 (SARS-CoV-2) infection itself. The incidence of SARS-CoV-2 positivity in children with neoplasms is low, and the majority of them have a mild or moderate course of infection. A physical examination of a child with a history of symptoms indicating cancer is crucial to proper evaluation.

It is essential to minimise the risk of detecting advanced childhood cancer to prevent children from experiencing neoplasms that are otherwise highly curable if diagnosed early, in addition to possible long-term complications and increased mortality.

Key words: COVID-19, pediatric oncology, children

Acta Haematologica Polonica 2021; 52, 3: 169-172

\section{Introduction}

The new coronavirus, severe acute respiratory syndrome coronavirus 2 (SARS-CoV-2), was first detected in Wuhan, China in December 2019 and caused the worldwide coronavirus disease 19 (COVID-19) pandemic announced by the World Health Organization (WHO) on 11 March, 2020. COVID-19 led to a critical situation in the healthcare system concerning patients suffering from cancer and chronic diseases. It had the effect of causing them anxiety and reluctance to seek immediate medical attention when required due to the fear of COVID-19's potentially acute and life-threatening states.

It is well known that the SARS-CoV-2 infection is more severe in the elderly and patients with comorbidities than in younger healthy people. The incidence and clinical course of SARS-CoV-2 in patients receiving anticancer or immunosuppressive therapy is still being studied, and they cannot be regarded as a low-risk group [1].

The consequences of COVID-19 in children are not directly life-threatening as most pediatric patients experience a mild or asymptomatic infection [2], but the indirect impact of the pandemic has become a real concern to healthcare professionals, especially pediatric oncologists and hematologists.

Based on recently published papers, the main issues concerning pediatric patients are the reluctance to seek care [2], suspended access to emergency facilities [2], and a significant decrease in regular in-person child healthcare visits [3]. These concerns have led to delayed referral of severe conditions [4], including children's neoplasms, that show a history of symptoms indicating cancer that

\footnotetext{
*Address for correspondence: Katarzyna Adamczewska-Wawrzynowicz, Department of Pediatric Oncology, Hematology and Transplantology, Poznan University of Medical Sciences, Szpitalna 27/33, 60-572 Poznań, Poland, e-mail: katarzynaadamczewska@wp.pl
}
PTHiT Copyright (c) 2021 The Polish Society of Haematologists and Transfusiologists, Insitute of Haematology and Transfusion Medicine. All rights reserved.


has been present for weeks. As is well-known, immediate diagnosis is still the most important prognostic factor in pediatric oncology and it must be emphasised that despite the COVID-19 pandemic, the proper clinical examination of a child should not be delayed and the warning signs of cancer must be carefully and promptly examined by family doctors and pediatricians.

The objective of this work was to describe the impact of the COVID-19 pandemic on pediatric oncology, especially its indirect aspect resulting in delayed cancer diagnoses.

\section{SARS-CoV-2 infection in children with neoplasms}

The spectrum of SARS-CoV-2 infection in children, accounting for less than $5 \%$ of all diagnoses, is generally milder than in adults $[5,6]$. Only a small number of pediatric patients need intensive care support and mechanical ventilation [6], and the majority of them have an asymptomatic-to-moderate course of COVID-19. It is well known that children under the age of 12 months are at risk of developing severe-to-critical infection [5]. Over the past few months, a vast number of publications have described a Kawasaki-like multisystem inflammatory syndrome which, due to its connection with the SARS-CoV-2 infection, has been called pediatric inflammatory multisystem syndrome temporally associated with SARS-CoV-2 (PIMS-TS, pediatric inflammatory multisystem syndrome temporally associated with SARS-CoV-2) [5]. Many studies concerning healthy children have been published, although the amount of data collected on SARS-CoV-2 infection in children with neoplasms is low. Some studies suggest a better outcome in immunocompromised patients due to lymphopenia and a lower risk of hyperinflammation [5]. A recently published paper on the epidemiology of SARS-CoV-2 infection in pediatric hematology in Poland described eight cases in which patients had an asymptomatic, mild or moderate clinical course that included neurological symptoms and pneumonia [1]. All patients were tested with real-time polymerase chain reaction (RT-PCR) on admission to the pediatric oncology ward in addition to healthcare staff working with the patients to avoid cross infection. The estimated incidence of SARS-CoV-2 infection in pediatric oncology patients was $0.5 \%$ in Poland [1]. According to the AIOEP/BFM, the incidence of infection oscillated around $0.74-0.82 \%$ and the overall death rate was $4 \%$, although in all fatal cases co-infections were present [1].

The recently published meta-analysis conducted by Dorantes-Acosta et al. [7] found no connection between COVID-19 and increased mortality in children with cancer, with overall survival of $99.4 \%$. Based on their systematic research, the authors stated that hematological neoplasms and solid tumors were not risk factors for a severe course of SARS-CoV-2 infection [7]. The low number of SARS-CoV-2 positive cases in children with neoplasms reflects the effectiveness of preventive principles that are carefully followed by both patients and caregivers, such as social distancing and the basic rules of infection control. These principles are continued in pediatric oncology clinics, and also include applying physical isolation, screening and providing limited access to the oncology ward by visitors [5].

Taking into consideration a small group of pediatric oncology patients with SARS-CoV-2 positivity with a mild-to-moderate course of infection, it is clear that the gravest danger arises from postponing or interrupting treatment of pediatric neoplasms, rather than from the virus per se [5]. During the months following the announcement of the COVID-19 pandemic, it became clear that the biggest danger to children with neoplasms or with a suspected oncological disease is reluctance to seek professional assistance caused by fear of the virus.

\section{Impact of COVID-19 on pediatric oncology}

Although the majority of pediatric patients with a SARS-CoV-2 infection do not experience life-threatening conditions, the infection has an indirect impact on other pediatric diseases, with neoplasms to the fore [2]. The main problem concerning pediatric oncology is delay in diagnosis and treatment [6]. Ciacchini et al. [2] reported that there had been a $76 \%$ decrease in admissions to the emergency department in the Cesare Arrigo Children's Hospital in Alessandria, Italy. Agostiniani et al. [3] claimed that Italian pediatricians observed a significant decrease in in-person child healthcare visits.

The fear of SARS-CoV-2 infection leads to the avoidance of caregivers to seek medical advice, thus preventing family doctors from noticing disturbing symptoms in a timely manner. Scientists from Italy found that more than half of respondents had recently used a search engine (with more than 40\% using Google) as their main resource to gain knowledge on their health problems and obtain medical advice online rather than appropriately consulting with pediatricians/physicians [2]. In Latin America, according to Vasquez et al. [8], there has been a huge decrease, of up to $60 \%$, in pediatric onco-hematology staff as a result of COVID-19 infection or quarantine.

Another concern emerging from the pandemic that is crucial in childhood cancer treatment is the shortage of blood product transfusions and transplants [9, 10], especially with short life products such as platelets and hematopoietic stem cells, due to difficulties in accessing unrelated donors and delays while waiting for negative SARS-CoV-2 tests [10]. It is important to remember that the time from noticing the first symptoms to the diagnosis of a pediatric neoplasm is the most important prognostic factor, and early implementation of treatment is crucial to recovery. 
Oncological diseases in children are dynamic and spread rapidly with just a few weeks of history of symptoms in most cases. Lazzerini et al. [11] described two cases of acute onset leukemia which occurred during Italy's national lockdown in 2020. The first patient complained of a very high fever, which was treated for seven days as an acute infection, while the second patient developed severe anemia with a hemoglobin level of $4.2 \mathrm{mg} / \mathrm{dL}$. This was unfortunately followed by respiratory distress and death due to delay in visiting the emergency department.

Another described case, which highlights the importance of physical examination, especially in children, was of a boy who was unable to defecate for more than seven days and was treated, after telehealth visits with his pediatrician, for functional constipation that in fact turned out to be Wilm's tumor (nephroblastoma) [11].

In all these cases, as reported by caregivers, the main reason for avoiding in-person visits to the emergency department was the fear of SARS-CoV-2 infection [11]. In their letter to the editor of Pediatric Blood \& Cancer, Ding et al. [12], from the U.S. Children's Hospital of Philadelphia and Lucile Packard Children's Hospital at Stanford, reported delays in children's cancer diagnoses emerging from the COVID-19 pandemic through describing five cases with severe consequences requiring immediate life-saving interventions, that were unfortunately unsuccessful in two children. They noted that from 2 March, 2020 to 6 April, 2020 there were no diagnoses of leukemia made, despite a stable five-year mean of 2.96 days between cases, with the longest no-new-diagnosis period from 2015 to 2019 amounting to 18 days [12]. Another disturbing fact was the necessity of referral to pediatric intensive care units and its prolonged use starting from the diagnosis, mainly due to cardiac arrest subsequent to acute lymphoblastic leukemia [12].

It is certain that sometimes clinical manifestation of children's neoplasms, including oncological emergencies, require immediate life-saving strategies, but specialists all over the world are observing disturbing increases in frequency during the COVID-19 pandemic. Offenbacher et al. [13] registered a drop in new solid tumor diagnoses from a mean number of 13.6 during March, April and May of 2015-2019 to no new diagnoses in March or May and only four new patients in April 2020, of which three were sarcomas with a long history of symptoms and detected metastasis, and the other was a brainstem glioma.

Carai et al. [4] noted a similar trend in March and April 2020 and described four patients with newly diagnosed brain tumors requiring life-saving treatment, with such decompensation only having been observed in $1 \%$ of cases in 2018 and 2019. The first patient, with vomiting lasting for 14 days accompanied by loss of consciousness, was finally diagnosed with an $8.5 \mathrm{~cm}$ parieto-occipital tumor with decerebration, resulting in bilateral fixed pupils and death [4]. In the second patient, a 5-month-old presenting with psychomotor regression of more than a month's duration, drowsiness, and neck deviation, was diagnosed with desmoplastic infantile astrocytoma [4]. Carai et al. [4] also described a case of progressive cachexia in an 8-year-old female with a 2-month history of polyuria and polydipsia, whose magnetic resonance imaging (MRI) revealed an infundibular lesion with metastasis and hemorrhage, which was confirmed to be a non-germinomatous germ cell tumor. Another female of the same age who experienced vomiting for more than three weeks with acute blindness and acute hydrocephalus on admission to the emergency department (ED), presented as the manifestation of a tumor in the fourth ventricle. Unfortunately, none of these patients were offered in-person medical visits and all were treated symptomatically after telehealth consultations with a family doctor [4].

The delays in proper clinical evaluation leading to a diagnosis of a tumor resulted in the death of one child and a life-long complication of blindness in the other [4]. Chiaravalli et al. [14] summarized the delay in pediatric solid tumor diagnoses in Italy during lockdown and registered only 16 (45.7\% of the expected number) new cases with two patients from Lombardy, compared to a total of 34-36 children between March and May in 2017, 2018 and 2019. The next eight weeks following lockdown brought 37 cases of pediatric solid tumors [14].

Healthcare professionals and scientists all over the world have noticed the collateral effect of the COVID-19 pandemic, impacting especially pediatric oncology and hematology because children's neoplasms are rare diseases, which without proper in-person clinical examinations and laboratory tests of initial symptoms, may be mistaken for SARS-CoV-2 infection or underestimated and taken as other milder conditions. As the era of telemedicine is emerging, this is becoming a grave concern to pediatric oncologists.

\section{Conclusions}

It must be understood that the risk of prolonging the diagnosis of other diseases, especially in the pediatric population, can be incomparably more threatening than the SARS-CoV-2 infection itself. The incidence of SARS-CoV-2 positivity in children with neoplasms is low, and the majority of them have a mild or moderate course of infection. Physical examination, in contrast to telehealth consultations, allows doctors to notice possible cancer manifestations such as pallor, hepatosplenomegaly, lymphadenopathy and neurological abnormalities that are crucial to proper evaluation. Another barrier that must be overcome is the delay in accessing medical care and postponing visits due to a caregiver's fear of SARS-CoV-2 infection, despite clear changes in the health and behavior of the child.

Anticipating further lockdowns, it is crucial to properly inform not only primary care physicians, but also parents 
and caregivers, about the possible signs of childhood cancer, especially with schools being closed, because teachers are often the first to notice disturbing symptoms.

It is essential to minimize the risk of detecting advanced childhood cancer to prevent children from experiencing neoplasms that are otherwise highly curable if diagnosed early, in addition to the possible long-term complications and increased mortality.

\section{References}

1. Styczyński J, Balwierz W, Wachowiak J, et al. Epidemiology and prevention strategies of SARS-CoV-2 infection in pediatric hematology and oncology centers in Poland. Acta Haematol Pol. 2020; 51(4): 253-257, doi: 10.2478/ahp-2020-0043.

2. Ciacchini B, Tonioli F, Marciano C, et al. Reluctance to seek pediatric care during the COVID-19 pandemic and the risks of delayed diagnosis. Ital J Pediatr. 2020; 46(1): 87, doi: 10.1186/s13052-02000849-w, indexed in Pubmed: 32600464.

3. Agostiniani R, Bozzola E, Staiano A, et al. Providing pediatric well-care and sick visits in the COVID-19 pandemic era: the recommendations of the Italian pediatric society. Ital J Pediatr. 2020; 46(1): 133, doi: 10.1186/s13052-020-00899-0, indexed in Pubmed: 32938482.

4. Carai A, Locatelli F, Mastronuzzi A. Delayed referral of pediatric brain tumors during COVID-19 pandemic. Neuro Oncol. 2020; 22(12): 1884-1886, doi: 10.1093/neuonc/noaa159, indexed in Pubmed: 32623465.

5. Bouffet E, Challinor J, Sullivan M, et al. Early advice on managing children with cancer during the COVID-19 pandemic and a call for sharing experiences. Pediatr Blood Cancer. 2020; 67(7): e28327, doi: 10.1002/pbc.28327, indexed in Pubmed: 32239747.

6. Wolfs TFW, Attarbaschi A, Balduzzi A, et al. COVID-19 - impact on childhood haematology patients. Hemasphere. 2020; 4(5): e465, doi: $10.1097 /$ HS9.0000000000000465, indexed in Pubmed: 32984769.

7. Dorantes-Acosta E, Ávila-Montiel D, Klünder-Klünder M, et al. Survival and complications in pediatric patients with cancer and COVID-19: a meta-analysis. Front Oncol. 2020; 10: 608282, doi: 10.3389/ /fonc.2020.608282, indexed in Pubmed: 33552980.

8. Vasquez L, Sampor C, Villanueva G, et al. Early impact of the COVID-19 pandemic on paediatric cancer care in Latin America. Lancet Oncol. 2020; 21(6): 753-755, doi: 10.1016/S1470-2045(20)30280-1, indexed in Pubmed: 32437662.

9. Sullivan M, Bouffet E, Rodriguez-Galindo C, et al. The CoVID-19 pandemic: a rapid global response for children with cancer from SIOP, COG, SIOP-E, SIOP-PODC, IPSO, PROS, CCI, and St Jude Global. Pediatr Blood Cancer. 2020; 67(7): e28409, doi: 10.1002/pbc.28409, indexed in Pubmed: 32400924.

10. Shoag JM, Barredo JC. The impact of COVID-19 pandemic on pediatric oncology. CellR4. 2020; 8: e2854.

11. Lazzerini M, Barbi E, Apicella A, et al. Delayed access or provision of care in Italy resulting from fear of COVID-19. Lancet Child Adolesc Health. 2020; 4(5): e10-e11, doi: 10.1016/S2352-4642(20)30108-5, indexed in Pubmed: 32278365.

12. Ding YY, Ramakrishna S, Long AH, et al. Delayed cancer diagnoses and high mortality in children during the COVID-19 pandemic. Pediatr Blood Cancer. 2020; 67(9): e28427, doi: 10.1002/pbc.28427, indexed in Pubmed: 32588960.

13. Offenbacher R, Knoll MA, Loeb DM. Delayed presentations of pediatric solid tumors at a tertiary care hospital in the Bronx due to COVID-19. Pediatr Blood Cancer. 2021; 68(2): e28615, doi: 10.1002/ /pbc.28615, indexed in Pubmed: 32725878.

14. Chiaravalli S, Ferrari A, Sironi G, et al. A collateral effect of the COVID-19 pandemic: delayed diagnosis in pediatric solid tumors. Pediatr Blood Cancer. 2020; 67(10): e28640, doi: 10.1002/pbc.28640, indexed in Pubmed: 32761998. 